\title{
Rubella Antibody Prevalence and Immunogenicity of Single Dose Rubella Vaccine Among 16 - 25 Years Girls from Bangladesh
}

\author{
Nessa $\mathrm{A}^{1}$, Tabassum $\mathrm{S}^{1}$, Akther $\mathrm{T}^{1}$, Sultana $\mathrm{N}^{1}$, Selim $\mathrm{S}^{2}$ \\ ${ }^{1}$ Department of Virology, Bangabandhu Sheikh Mujib Medical University, Dhaka, Bangladesh \\ ${ }^{2}$ Department of Endocrinology, Bangabandhu Sheikh Mujib Medical University, Dhaka, Bangladesh \\ e-mail: afzalunnessa@yahoo.com
}

\begin{abstract}
Rubella virus infection during pregnancy is an important cause of blindness, deafness, congenital heart disease, and mental retardation of the foetus. Multiple sero-surveys of rubella antibody among reproductive aged females of Bangladesh showed that 20-30\% of them remain susceptible to rubella as rubella vaccination is yet not included in our national immunization program for adolescent and adult girls. The present study was designed to conduct a sero-survey among unmarried girls of 16 to 25 years to assess their serological status in terms of natural rubella infection and vaccinate the rubella susceptible individuals with a single dose of rubella vaccine to evaluate the immunogenicity of the vaccine. A total of 344 randomly selected unmarried, apparently healthy college students were enrolled and investigated for rubella $\operatorname{IgG}$ and IgM antibodies at the Department of Virology, Bangabandhu Sheikh Mujib Medical University (BSMMU), Dhaka. Girls who were found to be negative for both rubella IgG and IgM were vaccinated with a single dose of rubella vaccine and tested for rubella antibody after four to six weeks of vaccination. Quantitative analysis of rubella IgG showed that a total of $68.87 \%$ study population had protective rubella antibody, $29.36 \%$ were susceptible to rubella and $1.74 \%$ experienced recent rubella infection. After vaccination, rubella antibody positivity was found to be $100 \%$ among 99 (98.02\%) of 101 vaccinated girls who were tested for anti rubella IgG. There was statistically significant increase $(p<0.001$, Paired $t$ Test $)$ in anti-Rubella IgG titres among pre-vaccination and post-vaccination sera. The findings of the study showed that it is mandatory to include young girls in the national immunization programme and immunize them with a single dose of rubella vaccine which was found to be $100 \%$ effective.
\end{abstract}

Keywords: Rubella antibody, Adolescent, Immunogenicity, Bangladesh

\section{Introduction}

Rubella as a clinical entity was first described by German authors in the mid eighteenth century. ${ }^{1}$ Although it is a mild exanthematous illness, because of the immense teratogenic potential of the virus, it can have disastrous consequences on the foetus if women of childbearing age contracted the infection during early pregnancy. Typically, rubella infection after birth is subclinical and occurs 14 to 21 days after exposure to the virus. In pregnant women, the virus can infect and replicate in the placenta. ${ }^{2}$ The outcome of foetal infection depends on the gestational timing of maternal rubella, but foetal infection can occur at any stage of pregnancy. ${ }^{3,4}$

The worldwide pandemic of rubella in 19621965 highlighted its importance. In the United States of America, alone there were an estimated 11,000 foetal deaths and 20,000 infants born with congenital rubella syndrome (CRS) during 1964 and 1965., 5 This pandemic stimulated the development of a safe and effective rubella vaccine. The World Health Organization (WHO) has advocated the use of rubella containing vaccines (RCV) in many countries recognizing the fact that CRS is a cause of preventable morbidity including childhood blindness and 
deafness, which in turn has life-long special health and social needs. Routine use of rubella vaccine is gradually resulting in the elimination of congenital rubella syndrome (CRS) in the developed world. The World Health Organization established goals to eliminate rubella and CRS in the WHO Region of the Americas by 2010 and in the Western Pacific Region for accelerated rubella control and CRS elimination by $2015 .^{7}$ In May 2012, including Bangladesh, the 194 Member States of the World Health Assembly resolved to endorse the Global Vaccine Action Plan (GVAP), which affirmed the elimination and control goals for measles and rubella by $2020 .^{8}$

Sustained vaccination strategy enabled the Americas to decrease rubella cases by $98 \%$, from $1,35,947$ in 1998 to 2,998 in 2006, and consequently the CRS incidence also decreased. ${ }^{9}$ In October 2012, Bangladesh replaced a singleantigen measles vaccine with a measles-rubella (MR) vaccine in the routine immunization schedule at nine months and at 15 years of age respectively. Unfortunately, adolescent and adult girls of child bearing age, who are presently at risk were not included in this programme. This may be due to inadequate information about the disease load as more than half of all cases are subclinical. Hence, active surveillance is required to determine the prevalence of the disease in Bangladesh, and to identify those who are vulnerable to infection and are at risk of giving birth to congenitally malformed babies. Thus, the present study was designed to determine the immune status for rubella among healthy unmarried girls of childbearing age and to identify the extent of the problem concerning rubella immunization with intent to motivate and vaccinate the susceptible individuals and assess the response to vaccine among them.

\section{Materials and methods}

Study population: Students from three Girls' Colleges of Dhaka city irrespective of socioeconomic strata were selected as study participants with written permission of the college authority. A total of 535 apparently healthy, unmarried girls between $16-25$ years of age with no history of childhood or recent rubella vaccination were interviewed to enroll them in this study. Ethical clearance was taken from the Ethical Committee of Bangladesh Medical Research Council (BMRC) prior to commencement of the study. Before data collection, informed written consent was also taken from the parents and/or respondents themselves. Socio-demographic, personal data and general information about rubella awareness were recorded through interview with a semistructured pre-tested questionnaire from the participants. With all aseptic precaution, $3 \mathrm{ml}$ of venous blood was collected from the study participants, labeled properly and after serum separation, preserved at $-20^{\circ} \mathrm{C}$ for further analysis.

Methods: Quantitative Rubella IgG and Rubella IgM antibody tests were performed by Chemiluminescence Enzyme Immuno Assay method using LIASON Rubella IgG and IgM kit (DiaSorin, Italy). All laboratory procedures were performed at the Department of Virology, BSMMU, Dhaka. The results were expressed as $\mathrm{IU} / \mathrm{ml}$ and $\mathrm{AU} / \mathrm{ml}$ for Rubella $\mathrm{IgG}$ and Rubella IgM respectively. Sero-positivity for Rubella $\mathrm{IgG}$ antibody was defined as $\geq 10 \mathrm{IU} / \mathrm{ml}$ ans for Rubella IgM as $\geq 25 \mathrm{AU} / \mathrm{ml}$, based on the cutoff values mentioned in the kit literature.

Rubella IgG and IgM antibody negative individuals were vaccinated with one dose $(0.5 \mathrm{ml})$ of rubella vaccine containing 1000 TCID50 of RA 27/3 strain (GlaxoSmithKline, Belgium) at the Vaccine Centre of the Dept. of Virology by subcutaneous route. Vaccine cards (in English) were issued to all vaccinated subjects and instruction for second visit after 4 weeks of vaccination was advised verbally and also mentioned in the cards. On their second visit, post-vaccination blood samples were collected and tested for Rubella IgG to assess the immunogenicity of the vaccine.

Data analysis: All obtained data were analyzed by computer-based software SPSS version 16 .

\section{Results}

Out of the 535 interviewed students, 346 gave written consent and were enrolled in the study. Of these, two sera were excluded due to hemolysis and finally 344 participants who met the inclusion criteria for the study were selected. The total participants were divided into five age 
groups at one year of age interval. Majority, $46.51 \%$ (160 out of 344) of the participants belonged to age group $16-17$ years. Out of the 344 participants, a total of 237 (68.89\%) was found to be positive for rubella $\mathrm{IgG}$ and 06 (1.74\%) for rubella IgM. However, 101 (29.36\%) were negative for both rubella IgG and IgM. The rubella immunity according to age groups observed that, younger girls were more susceptible to rubella infection than older age groups. A total of six girls in this study were positive for rubella IgM antibody indicating that these girls were recently infected with natural rubella infection (table-I).

Table-I: Distribution of Rubella IgG antibody in different age group of study subjects.

\begin{tabular}{|c|c|c|c|c|}
\hline $\begin{array}{c}\text { Age Group } \\
\text { (yrs) }\end{array}$ & $\begin{array}{c}\text { IgG Positive } \\
(\%)\end{array}$ & $\begin{array}{c}\text { IgM Positive } \\
(\%)\end{array}$ & $\begin{array}{c}\text { Negative } \\
(\%)\end{array}$ & Total \\
\hline $\begin{array}{l}16-17 \\
(n-160)\end{array}$ & $110(68.75)$ & $3(1.87)$ & $47(29.37)$ & 160 \\
\hline $\begin{array}{l}18-19 \\
(n-101)\end{array}$ & $67(66.33)$ & $2(1.98)$ & $32(31.68)$ & 101 \\
\hline $\begin{array}{c}20-21 \\
(\mathbf{n}-34)\end{array}$ & $24(70.58)$ & 0 & $10(29.42)$ & 34 \\
\hline $\begin{array}{c}22-23 \\
(n-32)\end{array}$ & $23(71.87)$ & $1(3.13)$ & $8(25.00)$ & 32 \\
\hline $\begin{array}{r}24-25 \\
(n-17)\end{array}$ & $13(76.47)$ & 0 & $4(23.53)$ & 17 \\
\hline $\begin{array}{c}\text { Total } \\
(\mathbf{n}-344)\end{array}$ & 237(68.89) & $6(1.74)$ & 101(29.36) & 344 \\
\hline
\end{tabular}

According to the study design, the susceptible girls $(n=101)$ who did not have any immunity to rubella were vaccinated and after four to six weeks of vaccination, on retesting of 99 participants, all (100\%) were found to be seropositive (figure 1)

Figure 1: Geometric mean titer (GMT) of pre-vaccination, natural Rubella infection and post-vaccination sample.

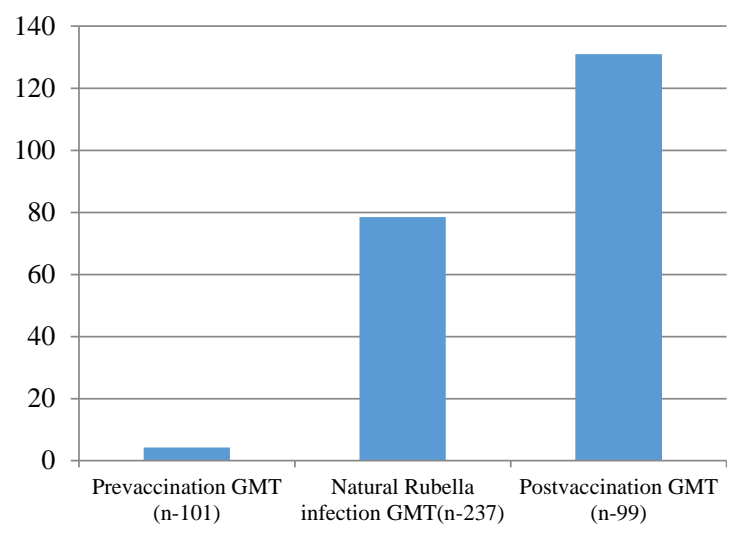

The geometric mean titre (GMT) of anti-rubella
$\mathrm{IgG}$ of the susceptible girls $(\mathrm{n}=101)$ before vaccination was $4.17 \mathrm{IU} / \mathrm{ml}$ (figure 1 ), which increased to $131 \mathrm{IU} / \mathrm{ml}$ after four to six weeks of vaccination. The difference between pre and post vaccination GMT of rubella antibody was statistically highly significant $(p<0.001$, Paired $t$ Test). All seronegative girls attained Rubella IgG antibody titre above the protective cut-off of 15 $\mathrm{IU} / \mathrm{ml}$ after vaccination (figure 2)

Figure 2: Scatter Diagram of Pre-vaccination and Postvaccination $\mathrm{IgG}$ titre among vaccinees.

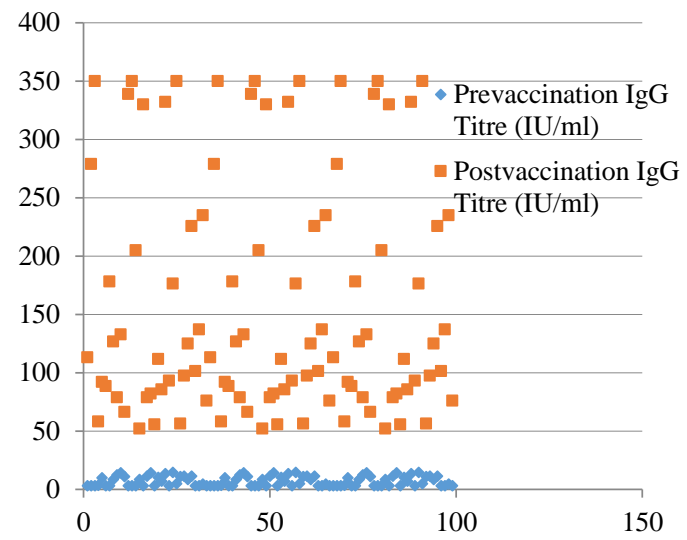

\section{Discussion}

Rubella virus infection has a public health importance as the disease poses a particular threat to the developing foetus if contracted during early pregnancy. In utero infection of the fetus may result in congenital deformities like cataract, congenital heart disease, deafness and mental retardation. ${ }^{1,10}$ Therefore, it is essential that girls develop immunity to rubella by the time they reach childbearing age to prevent such hazards. Immunity against rubella infection can be achieved by giving rubella vaccine which is safe, efficacious and cost effective. Since the licensure of the rubella vaccine in 1969, immunization programs have already had a major impact on the epidemiology of rubella in many developed countries. A review of published work sponsored by the WHO in 1996 reported an incidence of CRS of 0.6-2.2 cases/1000 live births during epidemics in developing countries, a rate similar to those of industrialized countries before vaccination. ${ }^{11}$ In January, 2000, the WHO held a meeting in Geneva directed towards prevention of CRS, particularly in developing countries and recommended to include rubella 
vaccine with measles and ensuring that the vaccination program cover children of both sexes and adult women. ${ }^{12}$

The present study was aimed to identify the rubella susceptible adolescent premarital girls of 16 to 25 years who were not included in our national program of immunization for rubella vaccination and to assess the effectiveness of a single dose of rubella vaccine on these vulnerable girls. Out of 344 girls, 237 (68.98\%) were found to be sero-positive for rubella IgG (table-I). The average seropositivity in the present study is comparable to our earlier study where rubella $\mathrm{IgG}$ prevalence was reported as $77.41 \%$ among 16 to 20 years of age. ${ }^{13} \mathrm{~A}$ similar trend was also observed in other studies among urban females of Dhaka city. ${ }^{14,15}$ The $68.98 \%$ rubella IgG positivity in our study indicates that a majority of the girls acquire protection against rubella by clinical or sub clinical natural rubella infection. All these previous studies including the present one reported a gradual increase of antibody prevalence with increasing age, which signifies the continuous exposure of individuals to rubella virus. The most important finding of the present study was that $29.36 \%$ of adolescent girls are still susceptible to rubella infection in our population. This imparts that a considerable number of these girls who stand a chance of early marriage, attain their child bearing age without acquiring natural rubella immunity against the disease. Non-immunized individuals are the candidates for natural rubella infection during outbreaks. Various epidemiological studies during outbreaks report that worldwide CRS rates occur among 0.7 to 2.2 out of 1000 live birth. ${ }^{6,11}$ Rubella is a highly contagious disease, and the overall incidence of disease among susceptible hosts at the community level during an epidemic range from 50 to $90 \%$, and is almost $100 \%$ contagious in closed populations. ${ }^{16,17}$ Humans are the only natural host of rubella virus, and virus-laden droplets from respiratory secretions of infected persons are the primary mode of transmission. The $1.74 \%$ IgM positivity observed in the present study indicates that every 2 out of 100 child bearing aged girls are infected continuously, and $29.36 \%$ susceptible girls in this study group are always in close contact with them. This is a matter of great concern since these girls are likely to get married any time in the coming years and will subsequently become pregnant. Therefore, it is important for them to have rubella immunity by this time and attention should be paid to them in order to prevent CRS.

Rubella vaccination is the most effective weapon against the crippling consequences of congenital rubella infection as the vaccine immunity is generally assumed to be lifelong. However, vaccine strain is the key factor influencing the persistence of rubella virus immunity. The most widely used strain for rubella vaccine is RA27/3. This strain generally induces higher antibody titres and produces antibody responses that mostly resemble natural infection. The resulting rubella immunity probably persists for a long time ( $>20$ years), and is therefore, the most widely used vaccine in the world. Several studies conducted abroad demonstrated a $100 \%$ seroconversion rate in children who had each been vaccinated with a single dose of RA 27/3 vaccine. ${ }^{18,19}$ According to the study design, rubella susceptible individuals $(n=101)$ in the present study were vaccinated with a single dose of MMR vaccine containing the RA 27/3 strain. Among the post vaccination cohort, 100\% subjects were found to be sero-protected with statistically significant rise of rubella antibody titer. This figure is comparable with other published literatures from abroad. ${ }^{20-22}$

The World Health Organization (WHO) established goals to eliminate rubella and CRS in the WHO Region of the Americas by 2010, the European Region by 2015, and in the WHO Western Pacific Region for accelerated rubella control and CRS elimination by 2015 . Sustained vaccination strategy enabled America to decrease rubella cases by $98 \%$, from $1,35,947$ in 1998 to 2,998 in 2006. Consequently, the CRS incidence had also decreased. In September 2013, the WHO Regional Committee for South-East Asia adopted resolution SEA/RC66/R5, with the goal of eliminating measles and achieving control of rubella/CRS by $2020 .{ }^{23}$ Among the member states of SEAR, Sri Lanka has already achieved the regional CRS control target of $<1$ CRS case per 100,000 livebirths and is progressing well to achieving zero endogenous transmission of rubella by $2020 .^{24}$ Sri Lanka was able to achieve this progress because rubella vaccination was included in their National Expanded Programme on Immunization (EPI) for women of reproductive age (16-44 years) since 1996, based on the evident CRS epidemics during 1994-1995. 
Since 2001, Sri Lanka introduced measles rubella (MR) vaccine for all children at the age of three years and subsequently, all girls and boys of 14 years were included in their national immunization programme since 2002 and 2003. Furthermore, to improve the population immunity to rubella infection, they conducted two catch-up vaccination campaigns with MR vaccine in 2003 and 2004 for 10-15-years and 16-20 years children respectively. ${ }^{24}$

Combined measles and rubella (MR) vaccine was included in National Expanded Programme on Immunization (EPI) in Bangladesh in September 2012, replacing the only-measles vaccine for children. $^{8}$ A large measles-rubella vaccination campaign for children aged nine months to less than 15 years was held in Bangladesh from January 25 to February 13. Regrettably, adolescent and adult girls of reproductive age who are presently at risk are not taken under consideration yet. Sri Lanka is very near to achieving the goal of zero endogenous transmission of rubella by 2020 , because they started rubella vaccination for reproductive aged female since 1996, gradually including all children, adolescent and adults females, as well as adolescent boys. The findings of the present study show that almost one third of the premarital 16 to 25 years females and 'would be mother' of the near future of Bangladesh remain rubella susceptible. To protect their foetus from the devastating effect of CRS, there is no alternative to rubella vaccination for reproductive aged females. To eradicate rubella from Bangladesh, vaccination of all infants may need 30-40 years and vaccination of all school girls up to 15 years will presumably eradicate CRS within $10-20$ years..$^{25}$

Therefore, to achieve the goal of CRS/Rubella control by 2020 in Bangladesh, the strategy of childhood vaccination alone does not seem adequate. National coverage of rubella vaccine for all reproductive aged females is essential to progress towards the goal of regional CRS control.

\section{Conclusion}

Findings of the present study revealed that all most one-third of adolescent and adult girls remain rubella susceptible. There is a great need to develop a comprehensive policy designed to protect mostly young adults and women of childbearing age in order to prevent congenital rubella infections.

Acknowledgments: The authors acknowledge with gratitude the financial support of Bangladesh Medical Research Council (BMRC) for this study. Furthermore, the authors are thankful to the Principals and the Management Committees of Shahjahanpur Girls College, Dhaka Women's College and University Women's Federation College of Dhaka city. Thanks are also extended to all participants for participants for their cooperation and enthusiastic participation.

\section{References}

1. Wesselhoeft C. Medical progress-Rubella. New Eng Journ Med. 1947; 236(25): 943-50.

2. Frij BJ, South MA, Sever JL. Maternal rubella and the congenital rubella syndrome. Clin Perinatol. 1988; 15: 247-57.

3. Miller E. Rubella in the United Kingdom. Epidemiol Infect. 1991; 107: 31-42.

4. Grillner L, Forsgren M, Barr B, Böttiger M, Danielsson L, De Verdier C. Outcome of rubella during pregnancy with special reference to the 17 th24th weeks of gestation. Scand J Infect Dis. 1983; 15: 321-25.

5. Assad F, and Ljungars EK. Rubella - World Impact. Rev Inf Dis. 1985; 7(Sup-1): S29-S36.

6. Robertson SE, Cutts FT, Samual R andDiaz-Ortega JL. Controll of rubella and congenital rubella syndrome (CRS) in developing countries, Part-2: Vaccination against rubella. Bull WHO. 1997; 75(1): 69-80.

7. Reef SE, Strebel P, Dabbagh A, Gacic-Dobo M, and Cochi S. Progress Toward Control of Rubella and Prevention of Congenital Rubella SyndromeWorldwide, 2009. Global Rubella Update, 2009. JID 2011:204 (1). S25

8. World Health Organization. EPI Surveillance Bulletin, Vol 15(1). Dhaka: World Health Organization; 2012.

9. Reef SE, Plotkin S and Jose F. Prepering for elimination of congenital rubella syndrome (CRS): Summary of a workshop of CRS elimination in the United States. Clin Inf Dis. 2002; 31: 85-95.

10. Ingalls TH, Babbott FL, Hampson KW and Gordon JE. Rubella, Its epidemiology and teratology. Am J Med Sci. 1960; 239: 363-83. 
11. Cutts FT, Robertson SE, Samuel R, Diaz-Ortega JL. Control of rubella and congenital rubella syndrome (CRS) in developing countries, part 1: burden of disease from CRS. Bull World Health Organ. 1997; 75: 55-68.

12. World Health Organization. Report of a meeting on preventing congenital rubella syndrome: immunisation strategies, surveillance needs. Geneva: World Health Organization; 2000.

13. Nessa A, Islam M.N, Tabassum S., Munshi SU, Ahmed M, Karim R. Seroprevalence of rubella among urban and rural Bangladeshi women emphasises the need for Rubella vaccination of prepubertal girls. Indian Journal of Medical Microbiology. 2008; 26 (1): 94-95.

14. Nahar $\mathrm{N}$ and Islam MN. Prevalence of rubella HI antibody in children and women in Dhaka. Bang Med J. 1987; 6: 1-5.

15. Ashrafunnessa, Khatun S, Islam MN and Chawdhury S. Seroprevalence of rubella antibodies among antenatal population attending a tertiary level hospital in Dhaka city. Bangladesh Med Res Coun Bull. 2000; 26(3):75-81.

16. Pollard RB and Edward AE. Epidemiologic survey of rubella in a military recruit population. Am J Epidemiol. 1975; 101: 431- 37.

17. Clark M, Schild GC, Boustred J, McGregor IA, Williams K. Epidemiological studies of rubella virus in a tropical African community. Bull World Health Organ. 1980; 58: 931-35.

18. Dunlop JM, Choudhury RK, Roberts JS, Bryett KA. An evaluation of measles, mumps, and rubella vaccine in a population of Yorkshire infants. Public Health. 1989; 103: 331-35.
19. Raut SK, Kulkarni PS, Phadke MA, Jadhav SS, Kapre SV, Dhere RM, et al. Persistence of antibodies induced by measles mumps- rubella vaccine in children in India. Clin. Vaccine Immunol. 2007; 14:1370-71.

20. Sharma H, Chowdhari S, Raina T, Bhardwaj S, Namjoshi G, Parekh S. Sero- Surveillance to assess immunity to Rubella and assessment of immunogenicity and safety of rubella vaccine in school girls. Indian J Community Med. 2010; 35 (1):141-44

21. Sharma H, Padbidri V, Kapre S, Jadhav SS, Dhere RM, Parekh SS, et al. Seroprevalence of rubella and immunogenicity following rubella vaccination in adolescent girls in India. J Infect Dev Ctries. 2011; 5(12):874-81.

22. Phalgune DS, Yervadekar RC, Sharma HJ, Dhere RM, Parekh SS, Chandak AD et al. Sero-surveillance to assess rubella susceptibility and assessment of immunogenicity and reactogenicity of rubella vaccine in Indian girls aged 18-24 years, Human Vaccines \& Immunotherapeutics. 2014; 10 (10): 2813-18.

23. Resolution of the WHO Regional Committee for South-East Asia. Measles elimination and rubella/congenital rubella syndrome control. In: Sixty-sixth session, South-East Asia Regional Office, New Delhi, India, 10-13 September 2013.

24. Gamage D, Galagoda G, Palihawadana P. Impact of rubella vaccination on elimination of congenital rubella syndrome in Sri-Lanka. WHO South-East Asia Journal of Public Health. 2015; 4 (2):189-96.

25. Plotkin SA. Rubella eradication. Vaccine. 2001; 19: 3311-19. 